CORRESPONDENCE

\title{
Anxiety and depression among Chinese adolescents during the COVID-19: an overestimation of the problem
}

(c) The Author(s) 2021

Translational Psychiatry (2021)11:624; https://doi. org/10.1038/s41398-021-01748-2

\section{DEAR EDITOR}

We read with interest the article by Chen and colleagues [1] which aimed to examine changes in depression and anxiety among Chinese adolescents during and after the initial COVID19 outbreak. These authors found that the prevalence of anxiety and depression significantly increased in Chinese adolescents after the initial outbreak. We commend the authors for addressing a pressing topic. However, we would call into question the validity of the results, and, further, the integral purpose of the article.

First, we believe the study design is not appropriate and poses several problems. This study involved the completion of two surveys: an initial survey completed by a sample of 9554 people and a second survey by 10,605 individuals. However, participants who completed both surveys $(n=6719)$ were removed. It is unclear why the authors decided to exclude such a large number of participants. Indeed, the rationale was not provided as to why they omitted the opportunity to conduct a longitudinal study using the two time points of collected data, rather than conducting two cross-sectional studies, which they opted for in this paper. Doing so would have allowed for more robust conclusions, as each participant would serve as their own control. In addition, within-subject designs are considered superior as they have greater statistical power [2].

In addition, excluding participants who completed both surveys also raises the question of statistical difference between the initial samples and the samples used in the analyses, after the exclusion of duplicate participants. Were these compared for the statistical difference? If so, why weren't the results reported?

Furthermore, Chen and colleagues [1] conclude that compared to the initial outbreak, "the prevalence of depression and anxiety significantly increased after the initial COVID-19 outbreak had remitted." However, the authors are comparing two different groups of people. Thus, stating that rates "increased" is problematic: rates may be higher, but the extrapolation of increased rates when examining two different sets of people is unwarranted. The authors treat the results as if they were a longitudinal study with temporal continuity. Besides, snowball sampling may be appropriate to examine relationships, but what about 'rates'? This is inappropriate.

Moreover, the authors present "prevalence" and "rates" of disorders (i.e., depression and anxiety), but this study exclusively used self-report measures as opposed to diagnostic interviews. The authors fail to report that self-report measures may not be used to diagnose disorders; further, they may substantially overestimate prevalence [3]. As such, caution should be employed when utilizing such terms as they would mislead readers. In addition, the cut-off scores the authors used for their measures (i.e., the Center for Epidemiologic Studies Depression Scale; CES-D, and the General Anxiety Disorder-7 scale; GAD-7) are extremely lenient and may result in inflation of the reported "rates" of anxiety and depression. For instance, the cut point of 4 on the GAD-7 was used in this study to identify adolescents with anxiety, whereas the original paper stated that a score of 10 or higher represents an adequate cut point for the identification of GAD-7 cases [4]. Other papers validating the GAD-7 among Chinese people with epilepsy and Chinese pregnant women utilized cut-off scores of 6 and 7 respectively $[5,6]$. Similarly, a cut-score of 15 on the CES-D was utilized to identify adolescents with depression in this study, whereas a systematic review of 28 studies proposed an optimal cut point of 20 [7]. Therefore, cut-off scores in this study appear to be too low, on top of selfreport measures already overestimating the prevalence, leading to a ringing of false alarms.

Further, we cannot help but notice that several elements are missing from the methods section. Indeed, there was no mention of how anything other than anxiety and depression was measured (i.e., the correlates of anxiety and depression that they explored). Measures of sleep duration, study duration, exercise duration, study efficiency, being concerned about entering a higher grade, are all absent.

All the points mentioned above bring into question the validity of their findings that appear quite an alarmist. Moreover, numerous other questions come up as we go through the paper. Importantly, were the assumptions for regression analyses tested? If so, why weren't they reported? Further, how did they manage data from participants who did not complete the totality of the survey (i.e., was the data discarded or corrected for)? What was the minimum completion rate accepted for participants to be included in the analyses? Additionally, it is stated in the paper that $46 \%$ of the initial sample were males, but what was the percentage of males in the final sample that was used in the analyses (i.e., after excluding participants who completed both surveys)? These questions stack up with the more fundamental problems in this paper.

Finally, we were wondering what the findings would have been, had different cut-off scores been used, and had a longitudinal design been used. We highly encourage the authors to re-run their analyses with these considerations in mind, or to make their data accessible for others to do so.

In sum, this paper tackled an important topic and a population that was heavily affected by the pandemic and whose mental health warrants investigation. However, considering the limitations, the results may be a misrepresentation of reality and an inflation of the gravity of the problem suggested. 


\begin{abstract}
Lara Kojok (D) ${ }^{1,2 \bowtie}$, Katie Bodenstein ${ }^{1}$, Marjolaine Rivest-Beauregard ${ }^{1,2}$, Quinta Seon (iD) ${ }^{1,2}{ }^{\prime}$ Ram P. Sapkota ${ }^{3}$ and Alain Brunet ${ }^{1,2}$ ${ }^{1}$ Department of Psychiatry, McGill University, Montréal (Québec), Montréal, QC, Canada. ${ }^{2}$ Douglas Mental Health University Institute, Montréal (Québec), Verdun, QC, Canada. ${ }^{3}$ Department of Psychology, University of Regina, Saskatchewan, Regina, SK, Canada. 凶email: lara.kojok@mail.mcgill.ca
\end{abstract}

\section{REFERENCES}

1. Chen X, Qi H, Liu R, Feng Y, Li W, Xiang M, et al. Depression, anxiety and associated factors among Chinese adolescents during the COVID-19 outbreak: a comparison of two cross-sectional studies. Transl Psychiatry. 2021;11:1-8.

2. Bellemare C, Bissonnette L, Kröger S. Statistical power of within and betweensubjects designs in economic experiments. IDEAS Working Paper Series from RePEc. 2014.

3. Thombs BD, Kwakkenbos L, Levis AW, Benedetti A. Addressing overestimation of the prevalence of depression based on self-report screening questionnaires. Can Med Assoc J. 2018;190:E44-9.

4. Spitzer RL, Kroenke K, Williams JB, Löwe B. A brief measure for assessing generalized anxiety disorder: the GAD-7. Arch Intern Med. 2006;166:1092-7.

5. Tong X, An D, McGonigal A, Park SP, Zhou D. Validation of the Generalized Anxiety Disorder-7 (GAD-7) among Chinese people with epilepsy. Epilepsy Res. 2016;120:31-6.

6. Gong $Y$, Zhou H, Zhang Y, Zhu X, Wang X, Shen B, et al. Validation of the 7-item Generalized Anxiety Disorder scale (GAD-7) as a screening tool for anxiety among pregnant Chinese women. J Affect Disord. 2021;282:98-103.

7. Vilagut $\mathrm{G}$, Forero $\mathrm{CG}$, Barbaglia G, Alonso J. Screening for depression in the general population with the Center for Epidemiologic Studies Depression (CES-D): a systematic review with meta-analysis. PLoS ONE. 2016;11:e0155431. https://doi.org/ 10.1371/journal.pone.0155431.

\section{AUTHOR CONTRIBUTIONS}

LK conceptualized and wrote the manuscript. AB and RPS helped with the conceptualization of the manuscript and provided thorough revisions throughout. $\mathrm{KB}, \mathrm{QS}$, and MR-B helped with the conceptualization of the manuscript and provided revisions on the final manuscript.

\section{COMPETING INTERESTS}

The authors declare no competing interests.

\section{ADDITIONAL INFORMATION}

Correspondence and requests for materials should be addressed to Lara Kojok.

Reprints and permission information is available at http://www.nature.com/ reprints

Publisher's note Springer Nature remains neutral with regard to jurisdictional claims in published maps and institutional affiliations.

(c) (i)

Open Access This article is licensed under a Creative Commons Attribution 4.0 International License, which permits use, sharing, adaptation, distribution and reproduction in any medium or format, as long as you give appropriate credit to the original author(s) and the source, provide a link to the Creative Commons license, and indicate if changes were made. The images or other third party material in this article are included in the article's Creative Commons license, unless indicated otherwise in a credit line to the material. If material is not included in the article's Creative Commons license and your intended use is not permitted by statutory regulation or exceeds the permitted use, you will need to obtain permission directly from the copyright holder. To view a copy of this license, visit http://creativecommons. org/licenses/by/4.0/.

(c) The Author(s) 2021 